\title{
De la crisis a las transiciones críticas en sistemas complejos: Hacia una actualización de la teoría de sistemas sociales
}

\author{
Aldo Mascareño*
}

\section{RESUMEN}

La teoría de sistemas sociales concibe la crisis como una autodescripción negativa de la sociedad moderna que mueve a la acción. Con ello, renuncia a entender el mecanismo generador de crisis sociales en sociedades complejas. Por medio de la teoría transdisciplinar de transiciones críticas, este artículo identifica el mecanismo de lock in como operación central en la producción de crisis complejas y presenta sus principales dinámicas. Esto mueve a tres aprendizajes en teoría de sistemas: la autopoiesis mantiene la continuidad del sistema pero también puede conducir a su colapso; la autodescripción como crisis funciona como un dispositivo que anuncia la proximidad de transiciones críticas; y la topología de transiciones críticas integra sociedad, naturaleza y tecnología. Con estas actualizaciones, la teoría de sistemas queda mejor preparada para enfrentar las crisis fuera de escala que afectan a la sociedad moderna en las últimas décadas.

\section{PALABRAS CLAVE}

Crisis, transición crítica, catástrofe, teoría de sistemas, Luhmann.

\footnotetext{
* Aldo Mascareño es PhD Sociología por la Universidad de Bielefeld, Alemania. Actualmente es Profesor Titular de Sociología de la Universidad Adolfo Ibáñez y del Centro de Investigación Modelos de Crisis. Artículo realizado en el marco del Proyecto Fondecyt 1181585. El autor agradece a Sebastián Raza y Mauro Basaure por la invitación a publicar en Theorein. Correo: aldo.mascareno@uai.cl
} 


\section{ABSTRACT}

Social systems theory conceives of crises as a negative self-description of modern society that moves into action. In so doing, systems theory renounces to understand the mechanism producing social crises in complex societies. By means of the transdisciplinary theory of critical transitions, this article identifies the lock in mechanism as the key operation producing complex crises, and presents its main dynamics. This elicits three insights for systems theory: the autopoiesis preserves the continuity of the system but also may lead to its collapse; the self-description as crisis works as a device announcing the proximity of critical transitions; and the topology of critical transitions integrates society, nature, and technology. By means of these improvements, systems theory becomes better prepared to deal with the off-scale crisis affecting modern society in the last decades.

\section{KEYWORDS}

Crisis, critical transition, catastrophe, systems theory, Luhmann. 
n las últimas dos décadas, el lado oscuro de la sociedad moderna se ha
mostrado con particular dramatismo y de manera cualitativamente distinta a décadas anteriores. Eventos que tradicionalmente hemos llamado crisis han adquirido robustez, extensión y periodicidad sin precedentes en la evolución social. La crisis financiera de 2008, por ejemplo, no se puede comprender cabalmente sino como un colapso sistémico por hipertrofia de complejidad: demasiada interconectividad (entre bancos y no bancos) y demasiada homogeneidad estructural (en los mecanismos de manejo de riesgo) deben ser siempre considerados anuncios de la cercanía de un cambio radical en cualquier constelación sistémica (Haldane y May, 2011). Asimismo, la crítica normativa que el colapso sistémico desencadena es, por un lado, relevante en sí misma y, por otro, produce consecuencias sistémicas profundas: con la crisis financiera se interrumpe el acceso al crédito, para ajustarse a la situación el Estado reduce el déficit fiscal limitando el gasto público, lo que conduce a una reducción de la confianza en las instituciones políticas democráticas y hace emerger liderazgos como el de Trump o procesos políticos como el Brexit (Walby, 2015).

Desde otra perspectiva, las migraciones masivas de Europa en 2015 muestran en qué medida sistemas políticos autoorganizados sobre premisas operativas distintas (en un caso con separación de poderes, en otro con concentración de poderes en la cúspide) pueden conducir a una confrontación transnacional deflacionaria en la que las posiciones se radicalizan. Pero en el mismo caso no se puede dejar de ver que los individuos no abandonan el futuro porque el presente parezca sin salida. Contra toda probabilidad de éxito, las precarias naves que cruzaron el Mediterráneo y las marchas de sirios (entre otros) por Hungría no solo encuentran motivación en la sobrevivencia, sino también en la realización de expectativas normativas que desafían la organización sistémica de las regiones receptoras. 
De un modo similar, los repentinos cambios en condiciones climáticas en distintas partes del mundo muestran la interconectividad de la spaceship Earth (Buckmeister, 2008) y, con ello, plantean la pregunta por el modo en que las operaciones sistémicas de los últimos siglos se acoplan con el mundo natural al punto de dar origen a una nueva era geológica llamada antropoceno (Davies, 2018). Pero no solo esto, pues si la relación sociedadnaturaleza transita de un loose a un tight coupling (Weick y Sutcliffe, 2015), entonces es esperable una protección de ese vínculo, por ejemplo, bajo fórmulas normativas como sustentabilidad/catástrofe (Billi, 2018): o se sigue la norma de la sustentabilidad o nos preparamos para un nuevo juicio final y el abandono el planeta -en el que seguramente ya no irán los justos, sino los futuros miembros de SpaceX.

Frente a acontecimientos de este tipo, me parece que la teoría de sistemas sociales tiene algunas ventajas sobre otros enfoques. En primer lugar, se trata de una teoría cuyo mensaje ha puesto énfasis en la formación de sistemas sociales autónomos cuyo control excede muchas veces las posibilidades humanas y las regulaciones que los propios sistemas crean para limitarse a sí mismos. En segundo lugar, la teoría nos ha dicho que los sistemas autónomos generan altas interdependencias y que, por tanto, de la combinación de autonomía e interdependencia cabe esperar más y no menos conflictos. En tercer lugar, la teoría nos ha hecho ver que el funcionamiento de sistemas es de carácter transnacional; por lo cual, regiones en las que se han logrado mayores niveles de coordinación intersistémica también pueden sucumbir por efecto de sus propias cegueras o por perturbaciones originadas en otras regiones donde las formas de coordinación son más bien negativas o antagónicas. Y en cuarto lugar, la teoría de sistemas también nos ha advertido que bajo condiciones de alta complejidad social, más que 
a contradicciones que se pueden resolver en un sentido u otro, hoy estamos expuestos a paradojas con las que hay que convivir.

Sin embargo, sea porque el concepto de crisis quedó indisolublemente ligado al de crítica desde la Revolución Francesa en adelante (Kosellleck, 1973), o porque la teoría crítica - la principal contradictora de la teoría de sistemas- adoptó esta diferencia como unidad de su práctica teórica y política, lo cierto es que la teoría de sistemas evitó sistemáticamente una teoría de la crisis de sistemas sociales. Desde que el propio Luhmann (1984) entendiera la crisis como una autodescripción negativa de la sociedad moderna que invita a la acción, hasta reflexiones recientes sobre la posibilidad de una teoría de sistemas como sociología crítica pero sin crisis (Esposito, 2017), el mecanismo de la crisis ha sido el tercero excluido de la relación entre sistema y entorno.

En este artículo quiero ofrecer una manera de aproximarse a las crisis en sistemas sociales complejos de las últimas décadas que pueda desentrañar las causas de su robustez, extensión y periodicidad. Al hacer esto, sustituyo el concepto de crisis por el de transición crítica, con lo cual recojo algunos últimos desarrollos sobre teorías de complejidad en investigaciones experimentales que son generalmente desconocidas para la sociología -en biología, ecología, física. Por actualización de la teoría de sistemas entiendo, entonces, el diseño de una subteoría dentro de un marco sistémico (la teoría de las transiciones críticas) para dar respuesta a un nuevo tipo de fenómenos complejos (las recurrentes e incontrolables crisis fuera de escala que afectan a la sociedad moderna en las últimas décadas).

El itinerario del artículo es el siguiente. En la primera sección expongo el modo en que la teoría de sistemas sociales ha comprendido el concepto de crisis. Pongo énfasis aquí en la noción de crisis como autodescripción negativa de la sociedad moderna. En la segunda sección introduzco 
la teoría de catástrofes de René Thom como antecedente de la teoría de transiciones críticas. En la tercera sección explico la teoría experimental de transiciones críticas tal y como esta ha sido desplegada en recientes teorías de complejidad. En la cuarta sección introduzco el concepto de transición crítica en la arquitectura de la teoría de sistemas sociales al ponerlo en relación con algunos de sus conceptos fundamentales. Finalmente, en la quinta sección, elaboro algunas conclusiones.

\section{La crisis de Niklas Luhmann: desde la desviación a la autodescripción}

El concepto de crisis aparece en varios libros y artículos de Luhmann, fundamentalmente en tres situaciones argumentativas: a) para referirse a acontecimientos empíricos relativamente conocidos o generalizables (crisis económica, crisis monetaria, crisis política, crisis de seguridad, crisis de la religión, crisis de la sociología); b) en el contexto de las discusiones con la teoría crítica y Habermas en particular; y c) en formas de argumentación teórica que, sin embargo, no se siguen sistemáticamente con posterioridad. Un tratamiento metódico del concepto no se realiza sino hasta 1984. En lo sucesivo, dejo de lado las referencias al concepto de crisis del tipo a) y b) e indago algunas de tipo c).

Una de las primeras ocasiones en las que Luhmann otorga algún posicionamiento teórico al concepto de crisis, este parece guiado por la diferencia normalidad/desviación -común en la sociología de Parsons, pero también en los análisis marxistas y de teoría crítica bajo la forma normalidad/ patología. En un artículo originalmente publicado en 1962 sobre el método funcional, Luhmann indica: 'Las crisis, los estados de excepción, las revueltas espontáneas y la catástrofes inesperadas son una ocasión favorable 
para el estudio no solo de esos eventos únicos, sino también, justamente, de las relaciones sistémicas normales que se interfieren con ellos' (Luhmann, 2005: 31). En este sentido, las crisis acontecen inesperadamente sobre un trasfondo de normalidad. Como lo discutiré en la tercera sección, al entender las crisis como transición crítica, es el propio 'funcionamiento normal' del sistema el que, internamente (no por una causa externa), produce situaciones críticas. Con ello, la distinción entre lo normal y lo patológico pierde aplicabilidad, pues - para ponerlo en esos términos- es la propia 'normalidad' la que produce la 'desviación'. Se trata, entonces, de una paradoja; no de una contradicción entre normalidad y desviación o entre normalidad y patología.

En análisis sobre el derecho a inicios de la década de 1970, Luhmann ya asocia la crisis a problemas de complejidad. Establece una correlación entre crisis e incremento de complejidad en áreas funcionales específicas. En esos casos: 'los desbalances constituyen una amenaza $[\ldots]$ que pone en peligro la estabilidad de la sociedad y desencadena desarrollos regresivos si es que no pueden formarse precondiciones complementarias dentro de un período corto de tiempo' (Luhmann, 1987: 165). La distinción normalidad/desviación continúa operando como distinción directriz. Sin embargo, la asociación entre crisis y complejidad anticipa algunos interesantes desarrollos futuros. El sistema puede reaccionar a crisis con cambios en la complejidad interna: 'El aumento de la variabilidad estructural posibilita un pre-desplazamiento y una reducción de los umbrales de crisis y, con ello, una ganancia en tiempo y oportunidades de reacción [...] El sistema se vuelve más sensible al entorno' (Luhmann, 1987: 243). Por otra parte —indica Luhmann— la complejidad del entorno no se deja a la crisis. Con el aumento de variabilidad interna, el sistema se ve enfrentado a más posibilidades desde las cuales seleccionar: 'En otras palabras, debe considerar la complejidad del entorno como 
relevante y poder manejarla con técnicas de selección mejoradas si es que quiere ahorrarse crisis' (Luhmann, 1987: 243). Si alguna posibilidad teórica hubo de incluir al tercero excluido crisis en la relación entre sistema y entorno en la teoría de sistemas era esta. Este camino, sin embargo, no es seguido por Luhmann.

No hay que olvidar que las décadas de 1970 y 1980 son relevantes en Alemania y Europa en relación al uso del concepto de crisis. La crisis del petróleo y la crisis ecológica comenzaron a tener presencia constante en el debate público de la época. Los problemas asociados al uso de la energía nuclear también entraron en escena, tanto que la discusión en torno a la crisis se convertiría -especialmente después de Three Mile Island y Chernobylen una discusión relativa a los riesgos permanentes de crisis producto de la complejidad tecnológica (Perrow, 1984; Beck, 1985). El problema se duplicaba, pues ya no se trataba solo de las crisis desatadas, sino también de las que acechaban en la complejidad y no podíamos anticipar (Luhmann, 2003).

En este contexto histórico, Luhmann (1984) ve que el concepto de crisis ha adquirido el carácter de una moda -seguramente por su uso inflacionario. Sociológicamente, en tanto, la crisis es entendida como un tipo especial de autodescripción de la sociedad mundial moderna denominada autodescripción negativa. Esta forma ya ha abandonado la distinción normalidad/desviación con la que originalmente se concebía la crisis y la transforma en un problema inmanente de la comunicación. Se trata, en este caso, de una autodescripción negativa porque la semántica de crisis coincide con la incapacidad de la sociedad moderna para describirse a sí misma como una unidad. Desde ninguna posición o interacción local es posible entregar una representación adecuada de esa sociedad en su totalidad: 'El sistema general es demasiado grande y demasiado complejo para ser inmediatamente 
entendible' (Luhmann, 1984: 59). La incapacidad de discernimiento de su complejidad es sustituida entonces por el concepto de crisis: este formula que 'las cosas andan mal' y no hace exigible demasiada explicación porque, además, supuesta la intransparencia de la complejidad, no puede darla. Por ello, la noción de crisis originalmente provoca alarma, llama la atención, y deriva la necesidad de una intervención práctica: la crisis invita a hacer algo — al menos a plantearse esa posibilidad.

En su artículo sobre el concepto de crisis, Luhmann (1984) se propone identificar teorías generales en las cuales tengan cabida tanto autodescripciones negativas como positivas de la sociedad moderna. Ellas son la teoría de la evolución, la teoría de la diferenciación sistémica y la teoría de sistemas autorreferenciales. No es el caso aquí reconstruir en qué consistirían las autodescripciones negativas y positivas en estas teorías. En todo caso, el argumento busca mostrar que la evolución no avanza hacia la perfección, que más diferenciación no es mejor que menos y que la autodescripción autorreferencial es simplificación en tanto condición de complejización. Más allá de esto, lo interesante del texto es que en varios pasajes Luhmann anuncia consideraciones relevantes para una teoría de la crisis en sociedades complejas -entendiendo crisis ya no como una autodescripción, sino como un fenómeno socio-histórico de carácter sistémico. Desataco las siguientes:

I. Si la descripción como crisis mueve a la acción, entonces el 'mejoramiento de las capacidades de nuestra sociedad para la autoobservación y autodescripción es una estrategia correspondiente e incluso preeminente' (Luhmann 1984: 63).

II. Si la sociedad moderna se organiza en sistemas sociales, entonces hay que esperar más y no menos crisis: 'Este [la crisis] es el resultado de 
precondiciones estructurales que prescriben alta autonomía, autoorganización y autorreproducción de elementos (autopoiesis) de subsistemas y altas interdependencias entre sistemas y entornos al mismo tiempo' (Luhmann 1984: 64).

III. Si la sociedad se diferencia en sistemas funcionales, entonces 'eso supone tener conciencia -incluso se puede entender como predicción- de crisis continuas, presiones de tiempo y de la necesidad de reestructuraciones que ni siquiera pueden pretender abrir las puertas para un futuro mejor' (Luhmann 1984: 65).

En estos tres mínimos pasajes, la crisis no se entiende solo como una autodescripción de la sociedad mundial moderna, sino como un problema principalmente asociado a las relaciones entre sistemas sociales autónomos. El problema de la crisis — desde este punto de vista— no está dentro de los sistemas mismos, sino que está en el hecho de que se requieran mutuamente para sostener sus propias autonomías. Puesto que cada sistema observa el mundo y se conduce bajo las directrices de su propia autonomía, y puesto que por ello cada sistema es cada vez más interdependiente de otros (porque requiere de lo que no puede hacer por sí mismo), entonces, en un escenario de diferenciación de ese tipo, es lógico esperar más crisis, conflictos, roces y fricciones.

Este concepto se mantiene hasta 1997 cuando se publica La sociedad de la sociedad —el último libro revisado por Luhmann. Ahí se puede leer, por ejemplo, que en la sociedad funcionalmente diferenciada ya no es posible suponer que el sistema más fuerte sea el que 'domina'; la probabilidad de fracaso global está integrada producto de las interdependencias. De ese modo, 'la extensión de la atención y la preocupación ya no pueden ser descritas por la metáfora de la 'fuerza', sino solo por la metáfora de la crisis' (Luhmann, 2007: 610). También se retoma la idea de crisis como desviación, pero ahora no como 
teorización propia, sino para describir el uso de la noción de crisis en teoría crítica: 'Los fenómenos críticos del presente se atribuyen [en la teoría crítica] a desarrollos equívocos que pueden corregirse' (Luhmann, 2007: 885). Con ello, para los críticos, la sociedad puede aprender de las experiencias consigo misma, con lo que la crisis tendería a desaparecer. Luhmann no niega que existan posibilidades de corrección, pero enfatiza en que toda corrección queda sometida a la evolución.

En síntesis, el uso que Luhmann da al concepto de crisis es más bien marginal. Esto puede deberse originalmente a una necesidad de diferenciación respecto de la teoría crítica, pero creo que también, luego del giro autopoiético en la década de 1980, se hacía más difícil concebir que la propia autopoiesis de los sistemas generara formas autoinmunitarias. Una teoría de las transiciones críticas puede ser una alternativa para encontrar una función de las crisis (y probablemente de la crítica) dentro del marco de la teoría de sistemas.

\section{La catástrofe como punto de partida}

Por distintas razones, la teoría de sistemas probablemente requiera de un concepto distinto para capturar teóricamente las situaciones de crisis en sistemas sociales complejos. En primer lugar, el propio Luhmann ya le asignó al concepto una posición como autodescripción negativa de la sociedad moderna que es coherente con los presupuestos de su teoría. $\mathrm{Si}$ esto es cierto, entonces - en segundo lugar- es consistente con la misma teoría de sistemas suponer que la comunicación por medio del concepto de crisis es una observación de primer orden que puede relevar aspectos socialmente problemáticos, pero que oculta operaciones que no tienen por qué ser evidentes o siquiera visibles para los actores que sufren las consecuencias (Roitman, 2014; Espósito, 2017). En tercer lugar, el concepto 
de crisis ha tenido múltiples variaciones semánticas a lo largo de la historia (Kosellleck, 2006), múltiples autodescripciones. Luhmann agrega otra más, pero no se hace cargo (no es su problema) de la pregunta si los sistemas tienen dinámicas críticas y cuál sería su mecanismo. Y en cuarto lugar, la identificación de la teoría crítica con el concepto de crisis, desde Marx en adelante, y la unidad entre crítica y crisis (Cordero, 2017), hablan en favor de una aproximación distinta —no porque la integración de esas perspectivas no sea posible o productiva (Cordero, Mascareño y Chernilo, 2017), sino porque no es necesario ver a Marx o a la teoría crítica con prismas sistémicos para comprenderla mejor. De lo que se trata es de comprender mejor las crisis sociales actuales en sistemas complejos sin asumir que alguien tiene el monopolio de 'la crisis'.

De este modo, propongo, en el marco de la teoría de sistemas sociales, continuar entendiendo el concepto de crisis como autodescripción negativa de la sociedad moderna, y denominar transición crítica al proceso objetual mediante el cual un sistema, una red, un ensamblaje de eventos naturales, tecnológicos y sociales produce condiciones de operación que conducen a una reestructuración significativa, muchas veces radical, extensa y generalmente rápida de los modos de organización y, con ello, de sus autodescripciones sociales —incluida la autodescripción como crisis.

Como lo observo en la sección siguiente, la teoría de transiciones críticas es un desarrollo reciente (desde la década de 2000 en adelante) en el amplio campo de estudios de sistemas complejos. Sus antecedentes, sin embargo, pueden rastrearse hasta la teoría de catástrofes de René Thom. Esto no es desconocido para Luhmann, aunque el término lo reserva para situaciones más bien excepcionales que consisten en 'el paso relativamente rápido de un principio de estabilidad a otro' (Luhmann, 2007: 519): el tránsito del primado de la segmentación a las sociedades centro-periféricas, o entre estas 
y la estratificación, y luego el tránsito a la diferenciación funcional. Cuando el reemplazo del principio de estabilidad 'lo cambia todo' (Luhmann, 2007: 519) es cuando se puede hablar de catástrofe. Es decir, para Luhmann, habrían existido tres catástrofes en el sentido de Thom. Lo demás sería metáfora.

El propio Thom, sin embargo, no ve las cosas de este modo. Thom (1975) distingue entre catástrofes ordinarias o regulares y catástrofes esenciales según las condiciones de aislamiento o interpenetración topológica en que tienen lugar. El límite es, en realidad, la calculabilidad: la mecánica planetaria es demasiado extensa y la cuántica demasiado pequeña para el cálculo de catástrofes. Pero entre esos extremos, las catástrofes son permanentes, tanto ordinarias como esenciales. Thom incluso lo formula a un nivel metateórico: 'La distinción entre puntos regulares y catastróficos es, obviamente, de algún modo arbitraria porque depende de cuán fina es la observación empleada. Se puede objetar, no sin razón, que cada punto es catastrófico a la luz de técnicas observacionales suficientemente sensibles. Esto hace de la distinción una idealización que se debe precisar por medio de un modelo matemático' (Thom, 1975: 38).

Siendo las cosas así, la estabilidad no es un estado de quietud. La comprensión del principio de estabilidad es abstracta, matemática, por tanto, universalmente aplicable a la clase de fenómenos que describe. Dicho en la abstracción de términos modelísticos: dada una familia de objetos $\mathrm{E}$ y un espacio de parámetros $S$, donde $E$ es un punto cercano a s en $S$ y t es cercano a s en S, el objeto Et tiene la misma forma que Es (Thom, 1975: 21 y ss). La estabilidad estructural o genérica es dinámica (metaestable, ultraestable, incluso se podría decir 'inestable'). En ella la propiedad E se mantiene; el objeto oscila en un basin of attraction con determinados límites de movimiento que no están determinados en S, sino en la relación ExS. E puede oscilar, pero oscila en un espacio de fase. 
La cuestión es distinta cuando se trata de morfogénesis. En un espacio B donde existen sistemas S y T es tiempo, BxT es el dominio donde tiene lugar el proceso. En ese dominio, un conjunto cerrado $\mathrm{K}$ cambia en cada punto su apariencia, de modo tal que 'el conjunto cerrado K será llamado el conjunto de puntos catastróficos del proceso' (Thom, 1975: 18). El pliegue de catástrofe es una bifurcación: 'ramificación (bifurcación) produce catástrofe' (Thom, 1975: 44). El espacio topológico que crean los atractores y sus basins constituye una gradiente dinámica separada por hipersuperficies (superficie de más de dos dimensiones). Cuando dos basins se interpenetran, la configuración puede ser compleja pero estable, produciendo, por ejemplo, una espiral en trayectoria cerrada. En tales casos se habla de lucha o competencia entre dos atractores. En situaciones como esta, el régimen del sistema varía de una fase a otra. Bajo condiciones de alta complejidad, sin embargo, cuando en el vecindario existen múltiples atractores, se rompe el principio de simetría (simetría en las causas conduce a simetría en los efectos) y la dinámica se vuelve caótica. Aquí, las catástrofes (bifurcaciones) ordinarias se ramifican en una catástrofe generalizada que no puede ser formalizada (Thom, 1975: 106). Pareciera ser que cuando Luhmann sostiene que solo el tránsito de un principio de estabilidad (tipo de diferenciación social) a otro puede ser denominado catástrofe, solo reconoce las catástrofes generalizadas de Thom; no observa las intersecciones más simples de dos (o más) basins of attraction que producen distintas (más o menos complejas) catástrofes ordinarias en un espacio topológico aún reconocible formalmente.

Es cierto que las extensiones sociales de la teoría de Thom (1990) no se han desplegado aún; pero que la estabilidad sea la norma y las catástrofes la excepción, como lo comprende Luhmann, simplemente es un error. Me parece que también tras esta comprensión subyace la diferencia 
normalidad/desviación que Luhmann hace a inicios de los años sesenta en su artículo sobre el método funcional que comenté en la sección 1. Dicho en otros términos, el principio de estabilidad es, para Thom, mucho menos 'estable' de lo que parece ser para Luhmann. Cuando Luhmann entiende que las catástrofes (seguramente catástrofes esenciales o generalizadas) solo existirían en el tránsito de un principio de diferenciación social a otro, presupone que no existirían catástrofes regulares bajo el predominio de un tipo de diferenciación. Esto, de algún modo, contradice la capacidad morfogenética de la sociedad que es clave en la teoría de la evolución del propio Luhmann (2007).

Es un hecho que el concepto de catástrofe elegido por Thom no ayuda mucho a la mejor comprensión del problema. Si hay tantos puntos catastróficos porque BxT es un dominio dinámico, entonces el mundo es un enjambre de catástrofes. El mismo Thom lo expone de este modo: 'Desde mi particular perspectiva, cada una de las discontinuidades en los fenómenos naturales deben ser tratadas como singularidades. El borde de esta mesa, el lugar donde la madera se transforma en aire es una superficie de separación, un dominio de catástrofe. Uno puede llamar a esto una catástrofe permanente respecto de la cual no es necesario poner atención [...]. Para mí, una catástrofe es una discontinuidad fenomenológica' (Thom, 2010: 23). Esta universalidad en el uso del concepto pudo haber sido lo que llevó a Luhmann a restringirlo al momento en que todas las relaciones sociales de una época comienzan a ser reemplazadas por otras nuevas que se sintonizan con el predominio de una forma de diferenciación social distinta. Solo hay que tener claro que esa interpretación luhmanniana no es la de Thom -aunque Luhmann la presente como si así fuera. Al hacerlo de este modo, Luhmann perdió la oportunidad de entender el mecanismo que hay 
tras el concepto de catástrofe de Thom. Esto es lo que logra la actual teoría de las transiciones críticas.

\section{La crisis como transición crítica}

La teoría de las transiciones críticas es tributaria de la idea original de Thom, pero lo es tanto (o tan poco) como, por ejemplo, Luhmann es tributario de Parsons. Ha sido desarrollada experimentalmente teniendo en consideración los más recientes avances teóricos y tecnológicos en matemáticas, computarización, ecología y otras disciplinas. La constatación inicial es simple y ha sido claramente expresada por Marten Scheffer, uno de los principales exponentes de esta teoría: 'sistemas complejos, desde el clima hasta los ecosistemas y la sociedad, pueden perder lentamente resiliencia hasta que una perturbación menor los puede empujar sobre un punto de inflexión' (Scheffer, 2009: 2). En este caso, la resiliencia tiene que ser entendida como la capacidad del sistema de recuperarse luego de una turbulencia y puede ser reconocida intuitivamente como un basin of attraction en el que la pendiente representa la dinámica del sistema sobre el que un atractor oscila (ver Fig. 1a y 1b).

$\mathrm{El}$ atractor no es ciertamente un punto de equilibrio o algo similar, sino una modalidad de operación. En el caso de la naturaleza, por ejemplo, un atractor es un rango de temperatura en el clima sobre el cual puede haber variaciones pero que en el mediano plazo se mantiene, o también una dinámica trófica en un ecosistema que permanece oscilando dentro de ciertos parámetros. En el caso de la sociedad, ejemplos pueden ser un patrón conductual grupal, una dinámica institucionalizada, una articulación recurrente de red, una forma relativamente establecida de hacer las cosas. La cuestión es que tanto en la naturaleza como en la sociedad los atractores no operan aisladamente unos de otros. Empíricamente 
siempre hay varios atractores gravitando en un determinado entorno (o landscape). En este sentido, 'una transición crítica tiene lugar cuando un sistema cambia de un atractor a otro' (Scheffer, 2009: 14) (ver Fig. 1c).

La relación de atractores con su landscape (con otros estados alternativos) puede acontecer de distintas maneras. Estilizadamente, la relación puede ser fluida. En ella el sistema logra procesar las variaciones de su entorno gracias a su metaestabilidad (es decir, a gracias a su capacidad adaptativa y suficiente variabilidad estructural) (Fig. 2a). En otros casos, el sistema es más bien insensible a las perturbaciones de su entorno hasta un cierto umbral crítico, luego del cual acontece una transición rápida hacia otro estado (Fig. 2b). En el tercer caso, el sistema puede desarrollar equilibrios alternativos y oscilar entre ellos de manera recurrente aunque no periódica. En estos casos, la transición puede ser mucho más violenta que en $2 b$, pues la curva se encuentra plegada sobre sí misma (Fig. 2c). 
Figura 1. Resiliencia y transición crítica ${ }^{1}$

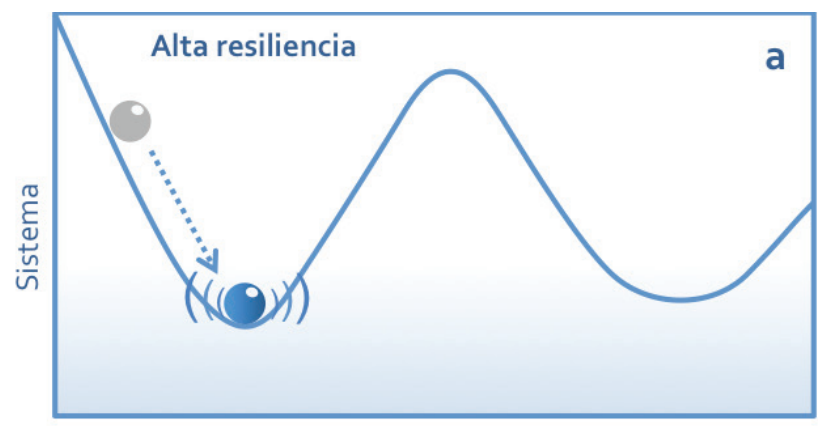

Condiciones
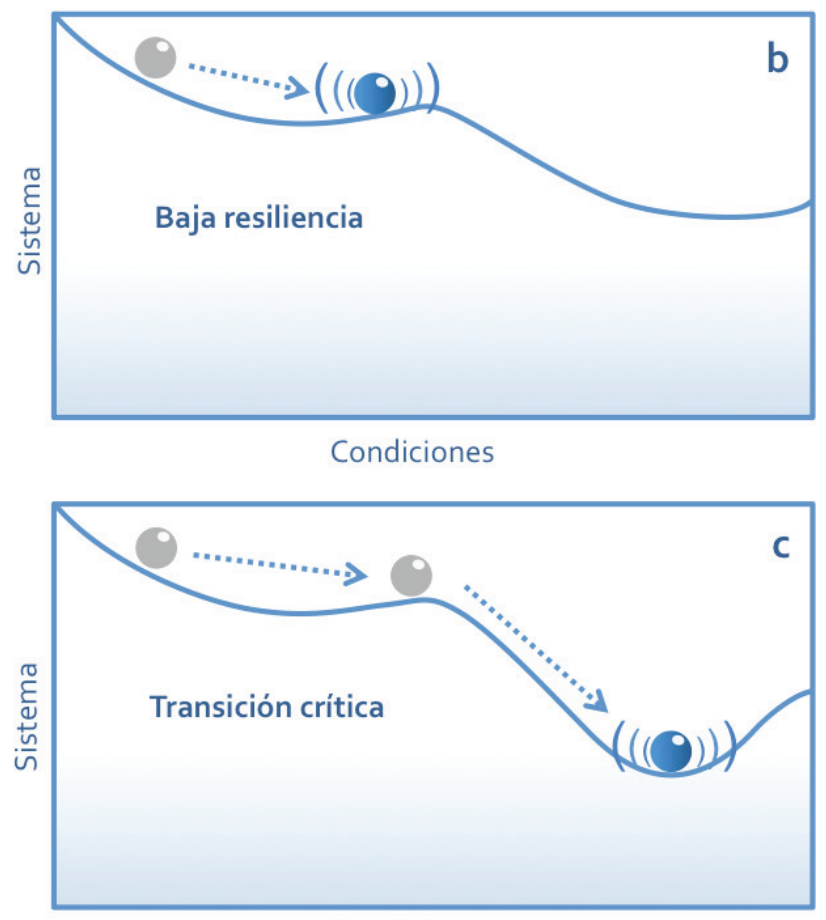

Condiciones 
Figura 2. Atractores en landscape ${ }^{1}$

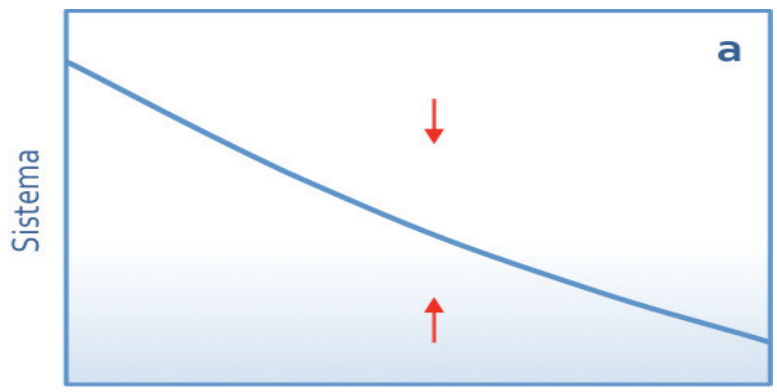

Condiciones

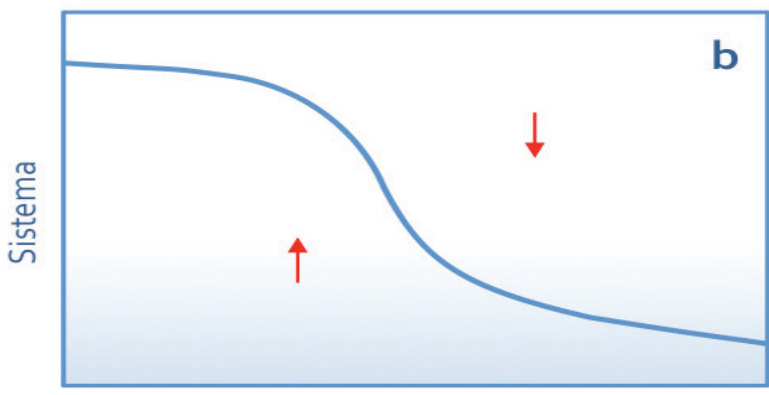

Condiciones

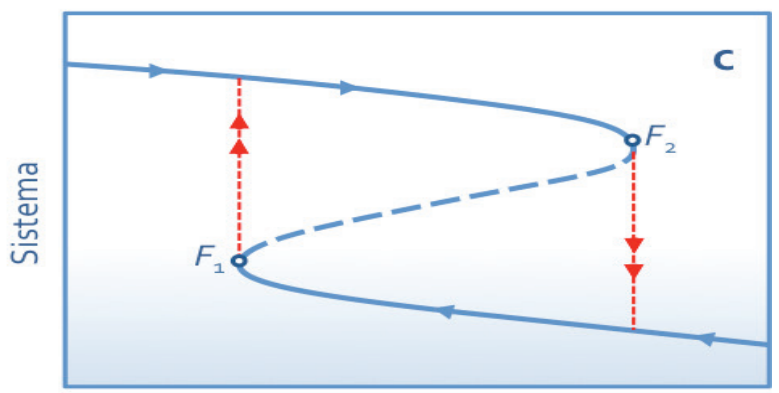

Condiciones

${ }^{1}$ Fuentes: Scheffer et al. 2001; Scheffer, 2009; Scheffer et al. 2012; Scheffer, 2016; van de Leemput et al., 2018 
A los casos $2 \mathrm{~b}$ y $2 \mathrm{c}$ se les puede denominar propiamente transición crítica. En ellos acontece un cambio de régimen que envuelve al landscape en su totalidad (sistema y entorno). Mientras en 2b (también en Fig. 1c) el sistema tiende a permanecer en su régimen alternativo, en $2 \mathrm{c}$ oscila entre ambos. El caso 2b, por ejemplo, puede representar un quiebre familiar, la disolución de una organización, un golpe de Estado exitoso, el colapso de una sociedad antigua - aquí puede incluso caber el ejemplo de Luhmann sobre la transición entre una forma de diferenciación y otra comentada en la sección anterior. El caso $2 \mathrm{c}$, en tanto, puede hacer referencia a situaciones sociales que son mucho más recurrentes cuando la complejidad (multiplicación e interconectividad de atractores) aumenta, por ejemplo, la oscilación de ciertos grupos en torno a los niveles de pobreza, la volatilidad electoral en varias sociedades contemporáneas, la sucesión de grupos que toman el poder de manera violenta en otras, las oscilaciones de los mercados financieros a nivel global y por supuesto sus grandes crisis, las variaciones en los temas de demanda de los movimientos sociales actuales, la competencia entre Estado de derecho y poderes particulares agrupados en torno al narcotráfico, la guerrilla, el crimen organizado. En todas estas situaciones (y otras), los cambios que tienen lugar son más bien rápidos y conducen de un régimen a otro; el sistema no puede transitar fluidamente desde F1 a F2 o viceversa; salta de uno a otro. En la literatura de sistemas complejos a estos momentos se les denomina de modos distintos pero equivalentes: bifurcation point (Sternberg, 2013), saddle node bifurcation (Shivamoggi, 2014), critical threshold (Scheffer, 2009), tipping point (Gladwell, 2002).

En las cercanías del umbral crítico el sistema pierde resiliencia, se hace cada vez más frágil y sensible a cambios estocásticos: una pequeña perturbación puede conducirlos hacia el régimen alternativo (Fig. 1c y 2c). 
En estos casos la histéresis del sistema —esto es, la dependencia del sistema de su memoria o su historia - juega un rol relevante. A mayor histéresis, la tendencia a regresar al régimen anterior es mayor (Scheffer et al., 2001). En ocasiones esto no podrá ser posible de manera rápida, sino solo en el largo o mediano plazo (por ejemplo, el retorno a la democracia después de un golpe de Estado exitoso), pero en otras la oscilación entre regímenes puede ser una situación recurrente (por ejemplo, las variaciones en mercados financieros, las oscilaciones en los temas que dominan la esfera pública, la volatilidad en la formación de opinión en Twitter, la movilidad alta en los flujos migratorios en períodos particulares, o la inestabilidad en las constelaciones de alianza en política nacional e internacional).

Si alguna posibilidad observaba Luhmann a la aplicación del concepto de crisis (transiciones críticas) más allá de la idea de autodescripción negativa, esta siempre se asociaba a la colisión entre lógicas sistémicas. Esto puede seguir manteniéndose. Sin embargo, los cambios de régimen en un landscape pueden acontecer tanto por la competencia de atractores como también - y principalmente - por las condiciones de operación interna del propio sistema propenso a una transición crítica — cuestión que la autopoiesis luhmanniana hace más difícil de visualizar. A esta operación interna del sistema que puede conducir a una transición crítica se le conoce como el mecanismo de lock-in.

Este mecanismo parece tener un comportamiento universal en sistemas complejos. A nivel celular conduce, por ejemplo, a la formación de funciones mayores por medio de un feedback positivo que refuerza y amplifica la trayectoria elegida, o incluso puede llevar a la muerte programada de células (apoptosis), por ejemplo, para la separación de los dedos en el embrión humano. A nivel de individuos, el mecanismo de lock-in puede conducir a estados depresivos o al cambio entre regímenes alternativos en 
escenarios maníaco-depresivos; también la interpretación de imágenes, de situaciones sociales o la recurrencia de patrones conductuales ilustran el mecanismo en el plano individual (Scheffer y Westley, 2007). Y a nivel social, los ejemplos pueden multiplicarse: las decisiones de inversión que no rinden lo esperado y se deben mantener porque el costo de abandonarlas es mayor, la perseverancia con un candidato político que no remonta en las encuestas porque el tiempo hasta las elecciones no permite una nueva campaña, la mantención dogmática de opiniones a pesar de las evidencias en contrario, el apego a determinadas semánticas normalizadas que pierden correlato estructural, la adhesión a conductas discriminatorias a pesar de cambios legislativos, la adaptación acrítica a formas de comportamiento grupal evidentemente contrarias a normas de civilidad humanas.

Estos mecanismos de lock-in han sido denominados de diversos modos en distintas disciplinas. En la teoría tradicional de sistemas se les llamó feedback loop (von Foerster, 1949), en teoría económica se habla de sunk-cost effects (Sutton, 2007), en investigaciones biológicas y psicológicas se les reconoce como el Concorde effect (Dawkins y Carlisle, 1976; Arkes y Ayton, 1999). La clave de este mecanismo es bien sintetizada por Scheffer: 'Este mecanismo de lock-in causado por una aparente adherencia autorreforzada a un modo de conducta tiende a promover inercia, una falta de responsividad a cambios en el entorno' (Scheffer, 2009: 245). En las situaciones en las que este mecanismo se despliega, el cambio en el sistema tiende a ser repentino (Fig. 2b y 2c) más que gradual (Fig. 2a); incluso lleva a situaciones de biestabilidad como las graficadas en Fig. 2c. La cuestión es, ahora, reconocer qué puede aprender la teoría de sistemas sociales de estas consideraciones. 


\section{Transiciones críticas y teoría de sistemas: tres aprendizajes}

Me parece que uno de los principales aprendizajes que la teoría de sistemas puede extraer de este análisis está en reconocer que la autopoiesis de la comunicación que sostiene el sistema es, a la vez, un mecanismo puede conducirlo a su colapso. La captura del sistema en un mecanismo de lock-in constituye una falta de reflexividad de la comunicación que limita o incluso impide su acoplamiento con el entorno (Cordero, Mascareño y Chernilo, 2017).

Es cierto que la autopoiesis no prescribe un único modo de funcionamiento sistémico: 'Para mantenerse abiertas a las irritaciones, las estructuras de sentido están elaboradas de tal manera que forman horizontes de expectativas donde se dispone de redundancias; es decir, repetición de lo mismo en otras situaciones' (Luhmann, 2007: 627). En concreto, la política puede funcionar con un gobierno conservador o con uno liberal, o la economía de mercado continúa en operación con alta regulación estatal o con un escenario desregulado; en ambos casos hay autopoiesis. Sin embargo, las redundancias tienen que poder ser seleccionables. Para ello, la autopoiesis no solo tiene que desarrollar capacidad autorreferencial; también tiene que disponer de heterorreferencia para la constitución de acoplamientos estructurales. Sin embargo, cuando la comunicación sistémica es capturada en un mecanismo de lock-in, se produce una reiteración irreflexiva de la autopoiesis que limita (incluso impide) la relación con el entorno. El lockin es una implosión de la reflexividad sistémica hacia una autorreferencia ciega que hace perder resiliencia y mueve al sistema hacia una transición crítica cuya violencia puede ser más alta o más baja dependiendo de cuán resistente sea la situación generada por la adherencia a un modo de conducta particular. 
En estos casos no hay perturbación externa; es la misma autopoiesis la que no logra resistir sus propios rendimientos. Aquel patrón conductual que sostiene el sistema es el que lo lleva a su colapso. Con ello, la autopoiesis se vuelve a la vez que un mecanismo de inmunidad sistémica que rechaza interferencias externas, un mecanismo autoinmunitario que se ataca y puede destruirse a sí mismo. Dicho en corto: robustez es fragilidad. Ejemplos de esto se pueden encontrar en distintas constelaciones, desde interacciones incrementales de violencia intrafamiliar hasta el colapso de sociedades antiguas por la explotación intensiva de recursos en un espacio ecológico específico (Tainter, 2017), o el colapso de sociedades modernas por la persistencia en un control jerárquico de la complejidad (Strayer, 1998), pasando por múltiples ejemplos organizacionales que es posible reconocer (Gladwell, 2002).

Generalmente, las transiciones críticas se caracterizan por períodos largos de incubación en los que se desarrolla el mecanismo de lock-in, un momento de propagación de las condiciones críticas y una situación de cambio de régimen o reestructuración (Mascareño, Goles y Ruz, 2016) que puede oscilar al régimen anterior dependiendo de la histéresis (Fig. 2c) oscilación o biestabilidad que, además, puede mantenerse por largo tiempo mientras ninguno de los regímenes alcance regularidad suficiente. Se puede pensar aquí en ejemplos de gran escala como la transición del esquema feudal al de los Estados nacionales, o en el de los Estados nacionales al de regiones transnacionales. Pero también se pueden considerar relaciones de pequeña escala, como la miserable oscilación de los conflictos de pareja previos a una separación (o a un reencuentro), o los períodos con direcciones interinas en distintas organizaciones, o las disputas de poder entre facciones al interior de partidos políticos. 
Un segundo aprendizaje de la teoría de sistemas tiene que ver con el estatuto de las autodescripciones negativas. Como lo sostuve en la sección 1, la crisis para Luhmann es una autodescripción negativa de la sociedad moderna que coincide con la incapacidad de esa sociedad para describirse a sí misma como unidad. El mundo es demasiado complejo para ser abarcado en autodescripciones unitarias (positivas) como felicidad, solidaridad, justicia, cohesión (Luhmann, 1997). Distintos espacios sociales generan sus propias variaciones al respecto — quizás si la única posibilidad que quede de autodescripción unitaria sean formulaciones paradójicas como la de unidad de la diferencia, de la diversidad, de la pluralidad. Pero si esto es así, las autodescripciones negativas como la de crisis, emancipación, pérdida de sentido, catástrofe, ingobernabilidad (Luhmann, 1984), no solo deben ser consideradas como una invitación a la acción, sino como una manifestación que tiene un correlato estructural directo.

En vez de suponer, como hace Luhmann, que la autodescripción llena un vacío de entendimiento acerca de las causas globales de los fenómenos con una invitación a la acción, parece sociológicamente más adecuado suponer que la autodescripción como crisis es una manifestación de la experiencia de los actores que la formulan. El correlato estructural de esto (que los actores no tienen por qué reconocer) son los mecanismos de lock-in que atrapan a los sistemas en una modalidad conductual que excluye a los afectados y que lleva al mismo sistema al colapso. Los actores pueden llamar a esto crisis; la teoría de sistemas puede denominarles, consistentemente con sus presupuestos, transiciones críticas.

La teoría experimental de transiciones críticas reconoce la existencia de dos mecanismos que anticipan la cercanía de ellas. Uno es el de recuperación lenta (critical slowing down); el otro es el de parpadeo (flickering). La recuperación lenta tiene lugar en las cercanías del umbral crítico (F1 o F2 
en Fig. 2c) y observa la tasa de recuperación del sistema ante perturbaciones menores: mientras más lenta es esa recuperación, más cercano es el umbral crítico luego del cual se produce la transición. Ejemplos donde esto puede ser observado o experimentado en situaciones sociales son el grado en que protestas masivas, movimientos sociales o formas de resistencia afectan a un sistema político, el tiempo en que una empresa se recupera de un paro sindical o de un impacto de mercado, el tiempo en el que un partido político se recobra de una derrota electoral o de un éxodo de miembros, la extensión del período en el que una institución (iglesia, empresa, grupo político) recupera la confianza luego de escándalos.

El parpadeo, en tanto, acontece en situaciones altamente estocásticas (en el que el evento próximo no está determinado por el anterior). Scheffer et al. (2012: 346) lo formulan del modo siguiente: 'Cuando un basin of attraction alternativo comienza a emerger, uno puede esperar que en entornos estocásticos los sistemas ocasionalmente salten [flip] a ese estado [...] Una varianza creciente puede reflejar tal cambio'. En estos casos el sistema anuncia momentáneamente un futuro alternativo: los alzamientos militares sofocados previo a un golpe militar mayor, las primeras migraciones de grupos pequeños antes de un flujo masivo, los escapes aún riesgosos de un sistema totalitario antes del colapso de sus fronteras, las empresas que entran en iliquidez antes de una corrida financiera global. No es que estos sistemas sean en sí mismos, ontológicamente, estocásticos, sino que sus grados de aleatoriedad se incrementan en la cercanía de los umbrales críticos. Por ello producen situaciones en las que, momentáneamente, teniendo en consideración Fig. 2c, se puede saltar de la rama superior a la inferior y volver a la superior aún bajo condiciones altas de histéresis generadas por el mecanismo de lock-in. 
Si estos dos mecanismos anteriores de anticipación de transiciones críticas tienen sentido en el ámbito de los sistemas sociales, la autodescripción negativa de crisis como manifestación de la experiencia de actores que vivencian situaciones de lock-in sistémico puede ser considerada un mecanismo específicamente social de anticipación de transiciones críticas - al que, además, convergentemente se le denomina crítica. En esto las expectativas normativas juegan un rol relevante. Ellas son más sensibles que las cognitivas a las situaciones de invariabilidad conductual. En base a la norma, los agentes pueden distinguir limitaciones o imposiciones recurrentes sobre el cumplimiento de sus estándares. Generalmente estas limitaciones o imposiciones son producidas porque el sistema opera atrapado en un mecanismo de lock-in (colusiones, privilegios de poder, hegemonías semánticas, autoritarismos incontestados) que restringen la contingencia y reducen posibilidades de selectividad. Puesto que la norma no está dispuesta aprender de esta situación, los agentes reaccionan con autodescripciones de crisis que contienen crítica normativa a las consecuencias y a la reproducción del lock-in respectivo.

Es cierto que la crisis puede ser una semántica inflacionaria que cuando se la utiliza para la descripción de situaciones sociales genera una red de significados preestablecidos, una especie de script que contiene normalidad y desviación, culpables y víctimas, alarma y emergencia, justificación de intervenciones y aceptación de regulaciones (Roitman, 2014). Sin embargo, una autodescripción crítica es también una operación de la sociedad, y como tal indica, directamente o indirectamente, un acontecimiento que tiene lugar en ella o que es relevante para ella. De este modo, la autodescripción negativa como crisis puede quedar incorporada dentro de la teoría de sistemas como una señal comunicativa, de los propios agentes, sobre la cercanía de una 
transición crítica — tanto como las medidas experimentales de recuperación lenta y parpadeo.

Un tercer aprendizaje que la teoría de sistemas puede hacer del análisis de transiciones críticas es reconsiderar la distinción fuerte entre sociedad, tecnología y naturaleza. Otra vez, esto no queda resuelto en el concepto de acoplamiento estructural, aunque tampoco se trata de que los mariscos y las redes pesqueras tengan alguna intencionalidad (Callon, 1984). Que los sistemas sociales estén estructuralmente acoplados a sus entornos naturales o técnicos y que por medio de ellos acepten determinadas influencias y limiten otras (Luhmann, 2007) es trivial. Este problema se hace particularmente complejo cuando se tienen en consideración desastres originados en la naturaleza (huracanes, terremotos, inundaciones, erupciones volcánicas) u otros tecnológicamente producidos (desde Chernobyl hasta Fukushima y las muertes que ya ha provocado la incipiente conducción autónoma). Que todo ello quede capturado en el concepto de riesgo (Beck, 1985) o más propiamente en la diferencia riesgo/peligro (Luhmann, 2003) no es suficiente, justamente porque la transición crítica ocurre cuando el riesgo dejó de ser una posibilidad y se transformó en desastre. Por así decirlo, la crisis es el fin del riesgo, o el colapso del riesgo da paso a la crisis.

Una transición crítica en la naturaleza (un terremoto, por ejemplo) actúa en muchos casos como una condición de borde de una transición crítica en la sociedad. Quizás solo baste recordar el rol que la historia de la filosofía le asigna al terremoto de Lisboa en 1775: el fin de la época del optimismo (Marquard, 1989). También Geoffrey Parker (2013) ha llamado la atención sobre la correlación que existe entre cambios abruptos en la temperatura en el siglo XVII y varias situaciones críticas que condujeron a llamar a este siglo el siglo de la crisis. Y seguramente no es necesario recordar los acontecimientos de las últimas décadas como los efectos del huracán 
Katrina en New Orleans, Louisiana y Mississippi, o los del tsunami de 2004 en Asia, o los del terremoto de 2010 en Chile.

En términos sistémicos, una transición crítica en la naturaleza genera una avalancha espontánea de información que fluye simultáneamente sobre distintos ámbitos sociales. Esto conduce a situaciones de alta inestabilidad sistémico-procesual expresada en saqueos, desórdenes públicos, colapso de centros médicos, de telecomunicaciones, de servicios. Ello coincide con la idea de catástrofe generalizada de Thom (1975). Lo que parece acontecer en estos casos es que con la avalancha simultánea de información, el escenario social se torna altamente estocástico, con lo que por un período - que puede ser más corto o más largo- el sistema social oscila caóticamente entre distintos atractores hasta la reconformación de algún régimen, generalmente el existente antes del colapso generalizado. En todo caso, esto siempre debe evaluarse sociológicamente por escalas: a gran escala es más probable una reestructuración elástica; a pequeña escala hay mayor probabilidad de reestructuraciones plásticas que no retornan al régimen anterior - por ejemplo, familias que pierden miembros o que sufren efectos traumáticos.

Cuando la teoría de sistemas distingue tan fuertemente entre sociedad, naturaleza y tecnología subvalora el continuum de materialidad que subyace a la comunicación. La inteligencia artificial, desde Siri hasta Sophia, pasando por Twitter, el machine learning y el big data marketing, introduce un alter ego no solo en la comunicación, sino también en la interacción. Incluso a este nivel, los humanos ya tienen que dar pruebas que no son máquinas introduciendo códigos para continuar con muchos procedimientos electrónicos. No hay duda que la comunicación social produce un orden emergente, que ella puede comunicar acerca de sí misma, de la naturaleza y de la tecnología. Pero por lo pronto, la tecnología está aprendiendo a hacer lo mismo, e investigaciones recientes parecen también encontrar equivalentes en la naturaleza (Vitti, 2013; 
Gorzelak et al., 2016). La sociedad puede seguir siendo sociedad, pero la comunicación deja de ser su monopolio en escenarios de alta complejidad.

Sin llevar por ahora este argumento al extremo, cuando se considera la universalidad de las transiciones críticas, la topología del landscape podrá incrementar su n-dimensionalidad al vincular sociedad, naturaleza y tecnología. Pero no habría que considerarlas como tres topologías distintas. La dinámica de la interrelación (o interpenetración) se puede reconocer en el mismo plano n-dimensional y formularse como landscape complejo. La comunicación queda recursivamente contenida en esa topología, básicamente porque la sociedad no reacciona en silencio cuando hay un desastre natural o tecnológico. Muy por el contrario, comunica sobre él y sobre sí misma en relación con él, lo que paradójicamente amplifica e incrementa las condiciones de crisis agregando más dimensiones a la topología de la transición crítica. Y asimismo, la comunicación traspuesta en acción se integra con el mundo natural por medios incrementales, como lo muestran las teorías del antropoceno (Davis, 2018), se integra también en interfaces sociotécnicas o biotécnicas de creciente carácter coevolutivo (Barfield, 2015) y, correspondientemente, la comunicación hace dependiente su propio futuro de estas relaciones de integración. Pasar por alto estos desarrollos porque la formulación teórica los diferencia es dogmatizar la teoría de sistemas.

\section{Conclusión}

Especialmente desde el cambio de siglo en adelante, situaciones críticas confusamente estructuradas denominadas wicked problems (Rittel y Webber, 1973), Hydra-crises (Hodge, 2013) u off-scale crises (Topper y Lagadec, 2013) han aumentado en robustez, extensión y periodicidad. La 
fuerte interconectividad en redes complejas (socio-técnico-naturales), la homogeneidad en sus modos de operación y la inmediatez en la propagación de rasgos, parecen crear un escenario distinto para que problemas locales escalen translocal o transnacionalmente.

Consistente con sus premisas, la teoría de sistemas ha posicionado la crisis como una autodescripción negativa de la sociedad moderna, como una semántica que mueve a la acción aun cuando no sea posible captar la causalidad tras los problemas experimentados. Con esto, la teoría de sistemas renuncia a comprender el mecanismo sistémico de las crisis - aunque en algunos pasajes de la obra de Luhmann es posible encontrar referencias a la crisis entendida como operación o como conflicto de lógicas sistémicas. Complementariamente, en la investigación experimental en ciencias de la complejidad, el nuevo modelo de transiciones críticas propone una forma de comprender crisis complejas y de reconocer sus modos de operación. El mecanismo de lock-in indica un comportamiento universal que explica transiciones rápidas en sistemas complejos y que la sociedad denomina crisis. Esto lleva a reconsiderar formulaciones sistémicas: a) la autopoiesis de la comunicación no solo produce continuidad del sistema, sino que también lo puede conducir a su colapso; b) la descripción como crisis y la crítica son indicaciones de la proximidad de transiciones críticas formuladas por los agentes a partir de sus propias experiencias con los mecanismos sistémicos de lock-in; y c) la diferencia sociedad-naturaleza-tecnología se interpenetra en la topología de las transiciones críticas, lo que integra operación social, natural y tecnológica en una red compleja n-dimensional y lleva a las crisis sociales actuales a un nuevo nivel de incontrolabilidad.

En una nota a pie de página en La sociedad de la sociedad, Luhmann sostiene: 'Las aplicaciones científico sociales de la teoría de las catástrofes de René Thom se han quedado en general detenidas en la pura metáfora' 
(Luhmann 2007: 519). Esto pudo haber sido cierto con Thom. Sin embargo, la metáfora se transforma en teoría cuando se explican los mecanismos de un determinado problema en el nuevo contexto. La integración de la teoría de las transiciones críticas dentro de la teoría de sistemas hace justamente esto en relación a la producción de crisis en sistemas sociales complejos, como este artículo lo ha mostrado. Alguna evidencia experimental también se puede aportar a ello (Mascareño, Goles y Ruz, 2016; Mora et al., 2016; Medina et al., 2017; Rengifo, Ruz y Mascareño, 2018). Esta señala rumbos posibles de investigación futura.

\section{Referencias}

Arkes, H. y Ayton, R. (1999) The sunk cost and Concorde effects: Are humans less rational than lower animals? Psychological Bulletin 125(5): 591-600.

Barfield, W. (2015) Cyborg-Humans: Our future with machines. Dordrecht: Springer.

Billi, M. (2018) Comunicación personal.

Buckminster, R. (2008) Operating Manual for Spaceship Earth. Zürich: Lars Müller Publishers.

Beck, U. (1985) Risikogesellschaft. Frankfurt: Shurkamp.

Callon, M. (1984) Some elements of a sociology of translation: domestication of the scallops and the fishermen of St Brieuc Bay. The Sociological Review 32(S1): 196-233.

Cordero, R. (2017) Crisis and Critique: On the fragile foundations of social life. Abingdon: Routledge.

Cordero, R., Mascareño, A. y Chernilo, D. (2016) On the reflexivity of crises: Lessons from critical theory and systems theory. European Journal of Social Theory 20(4): 511-530. 
Davis, J. (2018) The Birth of the Anthropocene. Oakland, CA: University of California Press.

Dawkins, R. y Carlisle, T. (1976) Parental investment, mate desertion and a fallacy. Nature 262: 131-133.

Esposito, E. (2017) Critique without crisis: Systems theory as a critical sociology. Thesis Eleven 143(1): 18-27.

Gladwell, M. (2002) The Tipping Point. New York: Back Bay Books.

Gorzelak, M., Asay, A., Pickles, B., y Simard, S. (2015) Inter-plant communication through mycorrhizal networks mediates complex adaptive behaviour in plant communities. AoB Plants 7: plv050.

Haldane, A. y May, R. (2011) Systemic risk in banking ecosystems. Nature 469 (7330): 351-355.

Hodge, B. (2013) The Hydra Paradox: Global Disaster Management in a World of Crises. Globalizations 10(3): 355-366.

Koselleck, R. (1973) Kritik und Krise. Frankfurt: Suhrkamp.

Koselleck, R. (2006) Crisis. Journal of the History of Ideas 67(2): 357-400.

Luhmann, N. (1984) The self-description of society: Crisis fashion and sociological theory. Journal of Comparative Sociology 25(1-2): 59-72.

Luhmann, N. (1987) Rechtssoziologie. Opladen: Westdeutscher Verlag

Luhmann, N. (1997) Globalization or world society: How to conceive of modern society? International Review of Sociology 7(1): 67-79.

Luhmann, N. (2005) Funktion und Kausalität (11-38). En Niklas Luhmann, Soziologische Aufklärung 1. Wiesbaden: VS Verlag.

Luhmann, N (2003) Soziologie des Risikos. Berlin: Walter de Gruyter.

Luhmann, N. (2007) La sociedad de la sociedad. México DF: Herder.

Marquard, O. (1989) Farewell to Matters of Principles. Oxford: Oxford University Press.

Mascareño, A., Goles, E. y Ruz, G. (2016) Crisis in Complex Social Systems. A Social Theory View Illustrated with the Chilean Case. Complexity 
21(S2): 13-23.

Medina, P., Goles, E., Zarama, R. y Rica, S. (2017) Self-Organized Societies: On the Sakoda Model of Social Interactions. Complexity ID 3548591. https://doi.org/10.1155/2017/3548591

Mora, F., Urbina, F., Cortez, V., y Rica, S. (2016) Around the Ising Model. Springer Proceedings in Physics 173.

Parker, J. (2013) Global Crisis. New Haven: Yale University Press.

Perrow, C. (1984) Normal Accidents. New York: Basic Books.

Rengifo, F. Ruz, G. y Mascareño, A. Managing the 1920's Chilean educational crisis. Plos One 13 (5): e0197429

Rittel, H. y Webber, M. (1973) Dilemmas in a General Theory of Planning. Policy Science 4: 155-169.

Roitman, J. (2014) Anti-Crisis. Durham: Duke University Press.

Schivamoggi, B. (2014) Nonlinear Dynamics and Chaotic Phenomena: An Introduction. Dordrecht: Springer.

Scheffer, M. (2009) Critical Transitions in Nature and Society. Princeton: Princeton University Press.

Scheffer, M. (2016) Anticipating societal collapse; hints from the Stone Age. PNAS 113(39): 10733-10735.

Scheffer, M. y Westley, F. (2007) The evolutionary basis of rigidity: Locks in cells, minds, and society. Ecology and Society 12(2): 36.

Scheffer, M., Carpenter, S., Foley, J., Folke, J. y Walker, B. (2001) Catastrophic shifts in ecosystems. Nature 413: 591-596.

Scheffer, M., Carpenter, S., Lenton, T., Bascompte, J., Brock, W., Dakos, V., van de Koppel, J., van de Leemput, I., Levin, S., van Nes, E., Pascual, M. y Vandemeer, J. (2012) Anticipating critical transitions. Science 338: 344-348.

Sternberg, S. (2013) Dynamical Systems. New York: Dover. 
Strayer, R. (1998) Why did the Soviet Union Collapsed? Understanding Historical Change. London: Routledge.

Sutton, J. (2007) Sunk Costs and Market Structure: Price Competition, Advertising, and the Evolution of Concentration. Cambridge, Mass.: The MIT Press.

Tainter, J. (2017) The Collapse of Complex Societies. Cambridge: Cambridge University Press.

Thom, R. (1975) Structural Stability and Morphogenesis. Reading, Mass.: W.A. Benjamin, Inc.

Thom, R. (1990) Semio Physics: A Sketch. Reading, Mass.: Addison-Wesley Publishing.

Thom, R. (2010) René Thom. Interviews with Emile Noël. Paris: IHES.

Topper, B. y Lagadec, P. (2013) Fractal Crises - A New Path for Crisis Theory and Management. Journal of Contingencies and Crisis Management 21(1): 4-16.

Van de Leemput, I., Dakos, V., Scheffer, M., y Van Nes, E. (2018) Slow recovery from local disturbances as an indicator for loss of ecosystem resilience. Ecosystems 21: 141-152.

Von Foerster, H. (1949) Cybernetics: Circular causal and Feedback Mechanisms in Biological and Social Systems. Transactions of the Sixth Conference. New York: Josia Macy Jr. Foundation.

Vitti, J. (2013) Cephalopod cognition in an evolutionary context: Implications for ethology. Biosemiotics 6(3): 393-401.

Walby, S. (2015) Crisis. Cambridge: Polity Press.

Weick, K. y Sutcliffe, K. (2015) Managing the Unexpected: Sustained Performance in a Complex World. Hoboke, NJ: Wiley. 
